\title{
Numerical simulation of orbitally shaken viscous fluids with free surface
}

\author{
Marco Discacciati ${ }^{3}$, David Hacker ${ }^{2}$, Alfio Quarteroni ${ }^{1,4}$, Samuel Quinodoz ${ }^{1, *, \dagger}$, \\ Stéphanie Tissot ${ }^{2}$ and Florian M. Wurm ${ }^{2}$ \\ ${ }^{1}$ MATHICSE, Chair of Modelling and Scientific Computing, Ecole Polytechnique Fédérale de Lausanne, Station 8, \\ CH-1015 Lausanne, Switzerland \\ ${ }^{2}$ Laboratory of Cellular Biotechnology, Ecole Polytechnique Fédérale de Lausanne, Station 6, CH-1015 \\ Lausanne, Switzerland \\ ${ }^{3}$ Laboratori de Càlcul Numèric (LaCàN) Escola Tècnica Superior d'Enginyers de Camins, Canals i Ports de \\ Barcelona (ETSECCPB) Universitat Politècnica de Catalunya (UPC BarcelonaTech) Campus Nord UPC - C2, E-08034 \\ Barcelona, Spain \\ ${ }^{4}$ MOX, Politecnico di Milano, P.zza Leonardo da Vinci 32, I-20133 Milano, Italy
}

\begin{abstract}
SUMMARY
Orbitally shaken bioreactors are an emerging alternative to stirred-tank bioreactors for large-scale mammalian cell culture, but their fluid dynamics is still not well defined. Among the theoretical and practical issues that remain to be resolved, the characterization of the liquid free surface during orbital shaking remains a major challenge because it is an essential aspect of gas transfer and mixing in these reactors. To simulate the fluid behavior and the free surface shape, we developed a numerical method based on the finite element framework. We found that the large density ratio between the liquid and the gas phases induced unphysical results for the free surface shape. We therefore devised a new pressure correction scheme to deal with large density ratios.

The simulations operated with this new scheme gave values of wave amplitude similar to the ones measured experimentally. These simulations were used to calculate the shear stress and to study the mixing principle in orbitally shaken bioreactors.
\end{abstract}

KEY WORDS: Navier-Stokes; multi-phase flows; free surface; finite elements; level set

\section{INTRODUCTION}

Orbitally shaken bioreactors (OSRs) have recently been expanded beyond simple shaken flasks to include disposable cylindrical bioreactors with nominal volumes of 200 and $2000 \mathrm{~L}$ for mammalian cell cultivation [1-4]. As a consequence, OSRs are now being considered as an emerging alternative to stirred-tank bioreactors for large-scale recombinant protein production with cultivated mammalian cells.

To optimize mammalian cell cultivation in OSRs, gas transfer, mixing rates and hydrodynamic stress must be understood and controlled because these are considered key to the successful application of any type of bioreactor. With mammalian cell cultures in bioreactors, achieving the maximum gas transfer and mixing is expected to require a high level of power input that would, in turn, increase hydrodynamic stress that could destroy cells. Thus, whereas efficient mixing minimizes concentration gradients of oxygen or $\mathrm{pH}$ [5], the conditions to assure this result need to be kept at the lowest

*Correspondence to: Samuel Quinodoz, MATHICSE, Chair of Modelling and Scientific Computing, Ecole Polytechnique Fédérale de Lausanne, Station 8, CH-1015 Lausanne, Switzerland.

$\dagger$ E-mail: samuel.quinodoz@epfl.ch 
level possible to limit damage to the cells. Hydrodynamic stress, however, is difficult to measure directly in bioreactors. Thus, computational fluid dynamic (CFD) simulations offer a way to assess this parameter and to study the hydrodynamic principles of OSRs.

Mathematically, the OSRs can be considered as a two-phase problem, where the position of the interface between the gas and the liquid phases is unknown. Nevertheless, the density of the two phases differs by three orders of magnitude and can profoundly affect the numerical results [6]. Extended finite element methods (XFEMs) have proved to be useful for dealing with interface problems in such situations $[7,8]$. However, their computational cost is higher than standard finite elements, and they require to modify available finite element codes and to develop novel preconditioning techniques [9].

In this paper, we studied a finite element method (FEM) to capture the interface discontinuities in a simple and efficient way. We used this mathematical technique to describe the behavior of the fluid in OSRs at different agitation rates. In particular, we focused on the shape of the free surface, the velocity field in the liquid, and the mixing behavior of the bioreactor.

After introducing the mathematical settings in Sections 2 and 3, we discuss the approximation in time and space in Section 4. In Section 5, we present several numerical results and comparisons with experimental data.

\section{SETTING AND DEFINITION OF THE PROBLEM}

Orbitally shaken bioreactors are usually made of two parts: a cylindrical container enclosing the cell culture and a rotary shaker (Figure 1(left)). The orbital shaking motion of the bioreactor can be represented as the sum of two rotations with opposite angular velocities $\omega$ : one around the axis of the cylindrical vessel and the other one around the center of the shaker (Figure 1(right)).

Mathematically, the fluid problem in an OSR can be considered as a two-phase problem, where the gas and liquid phases are separated by a free surface whose position is an unknown of the problem. The computational domain $\Omega$ representing the container depends on time and is composed of two parts: $\Omega_{\text {liquid }}$ and $\Omega_{\text {air }}$ corresponding to the liquid and gas phases, respectively (Figure 2 ). The two phases are separated by the free surface $\Gamma$ (Figure 2).

We assume that both the liquid and gas phases are incompressible Newtonian fluids. Thus, we can model them using the Navier-Stokes equations:

$$
\begin{gathered}
\rho_{a}\left(\partial_{t} \mathbf{u}_{a}+\left(\mathbf{u}_{a} \cdot \nabla\right) \mathbf{u}_{a}\right)-\nabla \cdot \mathbf{T}\left(\mathbf{u}_{a}, p_{a} ; \mu_{a}\right)=\mathbf{f}_{a} \quad \text { in } \Omega_{\text {air }} \\
\nabla \cdot \mathbf{u}_{a}=0 \quad \text { in } \Omega_{\text {air }} \\
\rho_{l}\left(\partial_{t} \mathbf{u}_{l}+\left(\mathbf{u}_{l} \cdot \nabla\right) \mathbf{u}_{l}\right)-\nabla \cdot \mathbf{T}\left(\mathbf{u}_{l}, p_{l} ; \mu_{l}\right)=\mathbf{f}_{l} \quad \text { in } \Omega_{\text {liquid }}
\end{gathered}
$$
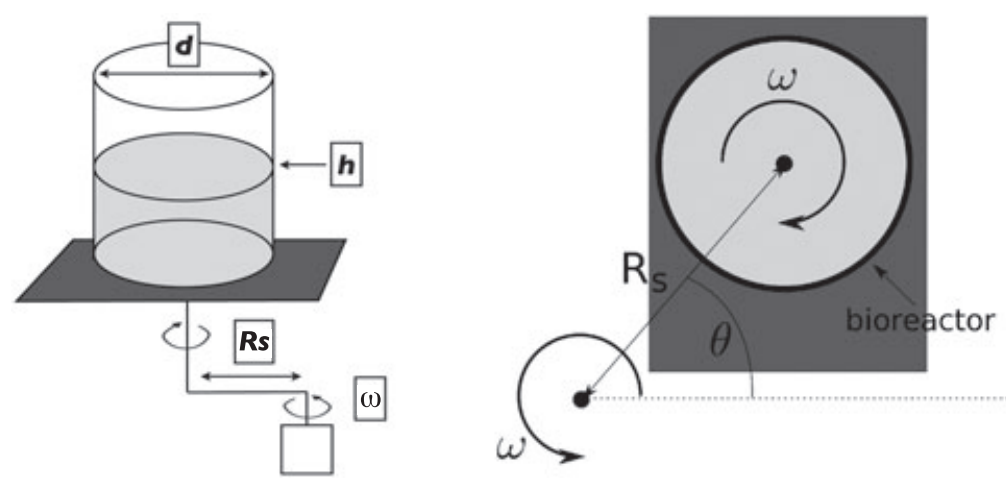

Figure 1. Side (left) and top (right) views of an orbitally shaken bioreactor (OSR); $d$ represents the inner diameter, $h$ the liquid height, $\omega$ the agitation rate, $R_{\mathrm{S}}$ the shaking radius, and $\theta$ the angle between the initial and current positions of the bioreactor. 


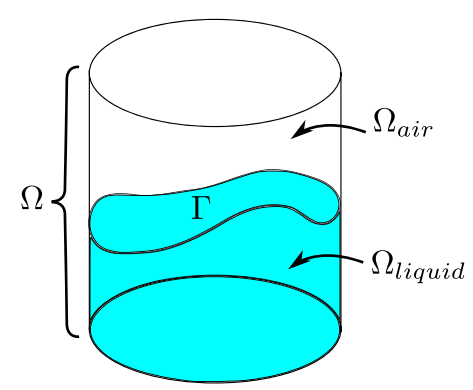

Figure 2. Schematic representation of the computational domain.

$$
\nabla \cdot \mathbf{u}_{l}=0 \quad \text { in } \Omega_{\text {liquid }}
$$

where $\mathbf{u}_{a}$ and $\mathbf{u}_{l}$ represent the velocity of the gas and the liquid, $p_{a}$ and $p_{l}$ their pressure, $\rho_{a}$ and $\rho_{l}$ their density and $\mu_{a}$ and $\mu_{l}$ their viscosity. $\mathbf{T}(\mathbf{u}, p ; \mu)=\mu\left(\nabla \mathbf{u}+\nabla \mathbf{u}^{T}\right)-p I$ is the Cauchy stress tensor and $\mathbf{f}_{a}$ and $\mathbf{f}_{l}$ represent the forces acting on the gas and the liquid. The interface $\Gamma=\Gamma(t)$ evolves according to the motion of the two phases.

We model our problem by writing the Navier-Stokes equations (1)-(4) in a fixed domain; the actual (imposed) motion of the shaking bioreactor will be represented by fictitious forces that have to be applied to the fluid.

We can decompose these fictitious forces into two components: a radial one to account for the centrifugal effects,

$$
\mathbf{f}_{r}=\rho R_{S}(\dot{\theta})^{2}\left(\begin{array}{c}
\cos (\theta) \\
\sin (\theta) \\
0
\end{array}\right)
$$

and a tangential one, describing the possible change in the agitation rate with a Coriolis type force

$$
\mathbf{f}_{t}=\rho R_{s} \ddot{\theta}\left(\begin{array}{c}
-\sin (\theta) \\
\cos (\theta) \\
0
\end{array}\right) .
$$

The total external force acting on the fluid (fictitious forces and gravity) can therefore be described as

$$
\mathbf{f}=\rho \mathbf{g}+\mathbf{f}_{r}+\mathbf{f}_{t}
$$

where $\mathbf{g}=(0,0,-g)^{T}$ is the gravitational acceleration, $R_{s}$ the shaking radius and $\theta=\theta(t)$ the angle between the position of the bioreactor and the horizontal axis (Figure 1).

In this work, we will assume that surface tension has a negligible effect on the flow. This assumption can be justified by considering the Eötvös number $E o$,

$$
E o=\frac{\left(\rho_{l}-\rho_{a}\right) g d^{2}}{\sigma}
$$

which is used to describe the relative importance of surface tension with respect to gravity, where $\sigma$ is the surface tension coefficient. In our case, the Eötvös number is of the order of 1000, indicating that surface tension can be neglected.

\subsection{Fluid flow boundary conditions}

Setting suitable boundary conditions is necessary to close the problem. However, the choice is not straightforward for problems with a free surface. Indeed, with reference to Figure 3 for the nomenclature, we use no slip conditions $\mathbf{u}=\mathbf{0}$ on $\Gamma_{\text {down }}$ and $\Gamma_{\text {up }}$ but not on $\Gamma_{\text {wall }}$, otherwise the free surface would not change on $\Gamma_{\text {wall }}$, a non-realistic situation. 


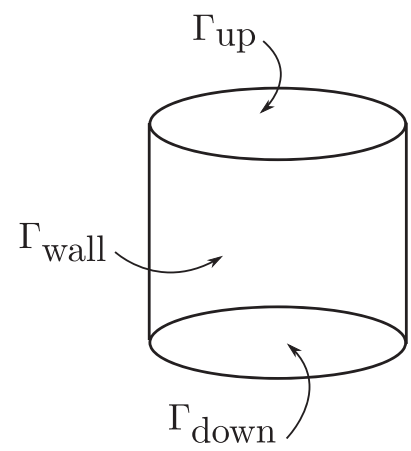

Figure 3. Parts of the boundary of the computational domain.

Several approaches have been proposed for this problem, see [10] for a review. We define $\mathbf{e}_{i}$ as the unit vector in the $i$ direction, $\mathbf{n}$ as the normal to the boundary $\Gamma_{\text {wall }}$ and $\tau$ as the tangential direction $\tau=\mathbf{e}_{z} \times \mathbf{n}$, and consider the following two possibilities:

- Vertical slip. It consists of imposing two Dirichlet conditions for the horizontal components of $\mathbf{u}$ :

$$
\mathbf{u} \cdot \mathbf{e}_{x}=0 \quad \mathbf{u} \cdot \mathbf{e}_{y}=0 \quad \text { on } \Gamma_{\text {wall }}
$$

and a Neumann condition along the vertical direction:

$$
(\mathbf{T}(\mathbf{u}, p ; \mu) \mathbf{n}) \cdot \mathbf{e}_{z}=0 \quad \text { on } \Gamma_{\text {wall }} \cdot
$$

- Tangential slip. In that case, only the no-penetration constraint is applied to the fluid:

$$
\mathbf{u} \cdot \mathbf{n}=0 \quad \text { on } \Gamma_{\text {wall }}
$$

(see [11] for the detail of the procedure to implement this kind of boundary conditions) whereas two Neumann conditions are applied on the other components of the velocity

$$
(\mathbf{T}(\mathbf{u}, p ; \mu) \mathbf{n}) \cdot \tau=0 \quad(\mathbf{T}(\mathbf{u}, p ; \mu) \mathbf{n}) \cdot \mathbf{e}_{z}=0 \quad \text { on } \Gamma_{\text {wall }} .
$$

In Section 5.5, we will study an alternative approach based on Robin boundary conditions on the stress on $\Gamma_{\text {wall }}$ together with the no-penetration constraint $\mathbf{u} \cdot \mathbf{n}=0$.

\section{TREATMENT OF THE FREE SURFACE PROBLEM}

To represent the free surface $\Gamma(t)$ at time $t$, we use the level set method [12] because it can naturally deal with large changes in the shape of the interface. We will also devise a pressure correction scheme that is especially adapted to that method.

For every time $t \geqslant 0$, the level set method consists in defining a scalar function $\phi: \Omega \rightarrow \mathbb{R}$ so that we can characterize the free surface as

$$
\Gamma=\{\mathbf{x} \in \Omega \mid \phi(\mathbf{x})=0\} .
$$

(The dependence of $\Gamma$ and $\phi$ on $t$ is understood.) In order to achieve a good numerical accuracy on the localization of the interface, the gradient of the level set function $\phi$ has to be moderate (neither too steep nor too flat); this is why $\phi$ is defined as being a signed distance function, that is, $|\nabla \phi|=1$. The location of the two fluids is then recovered by evaluating the sign of the level set function.

To move the interface $\Gamma$, we have to solve a pure transport equation given by

$$
\partial_{t} \phi+\mathbf{u} \cdot \nabla \phi=0 \quad \text { in } \Omega
$$

where $\mathbf{u}$ is the velocity of the fluid. However, this temporal evolution does not preserve the property that $\phi$ is a signed distance. A reinitialization procedure has to be used in order to recover this property. 
The main concern when one uses only the level set method (instead of coupling it with volume of fluid [13] or particle methods [14]) is volume conservation. As a matter of fact, because the advection process does not conserve the volume at the discrete level, and that the reinitialization moves slightly the interface position, the volume occupied by the two phases might change. This is a critical issue for long time simulations, as with time, the differences in the volume accumulate, and therefore, the final volumes might be far from the initial ones.

To alleviate this problem, so as to achieve physically realistic simulations, we used a variant of the level set correction proposed in [15]. When the exact volumes of the phases are known, this method consists in updating after each time step the level set function with the correction

$$
\phi(\mathbf{x}) \leftarrow \phi(\mathbf{x})+c \quad \forall \mathbf{x} \in \Omega
$$

where $c$ is a constant over $\Omega$ given by

$$
c=\frac{V_{\text {exact }}-V(\phi)}{|\Gamma|}
$$

where $V_{\text {exact }}$ is the exact volume of the fluid represented by positive signs of the level set function, $V(\phi)$ the volume of the fluid represented by positive signs of the level set function using the current values of the level set function $\phi$, and $|\Gamma|$ is the measure of the interface.

When applying this correction, we observed oscillations in the volume around the exact value. Therefore, we chose to use a relaxation parameter $\alpha$ in the correction of the level set function (11):

$$
\phi(\mathbf{x}) \leftarrow \phi(\mathbf{x})+\alpha c \quad \forall \mathbf{x} \in \Omega
$$

where $c$ is defined as mentioned earlier, whereas $\alpha$ is a real parameter that can be set to 1 to retrieve the original correction. However, numerical experiences showed that using a value of $\alpha$ equal or close to 1 results in oscillations of the volume around the exact value. Because the value of $\alpha$ must anyway be large enough to compensate for the numerical loss, we found $\alpha=0.5$ to be a good tradeoff.

\section{NUMERICAL APPROXIMATION IN TIME AND SPACE}

The Navier-Stokes equations and the level set method have been coupled to provide a model for the fluid flow problem with free surface. More precisely, the coupling occurs by defining a variable viscosity $\mu$ and density $\rho$ for the fluid in the Navier-Stokes equations on the basis of the sign of the level set function:

$$
\rho(\mathbf{x})=\left\{\begin{array}{cl}
\rho_{\text {liquid }} & \text { if } \phi(\mathbf{x}) \geqslant 0 \\
\rho_{\text {air }} & \text { if } \phi(\mathbf{x})<0
\end{array} \quad \mu(\mathbf{x})=\left\{\begin{array}{cc}
\mu_{\text {liquid }} & \text { if } \phi(\mathbf{x}) \geqslant 0 \\
\mu_{\text {air }} & \text { if } \phi(\mathbf{x})<0
\end{array}\right.\right.
$$

Conversely, once solved, the Navier-Stokes equations provide the velocity $\mathbf{u}$ of the fluid that is used to advect the level set function.

This problem is non-linear because of the intrinsic non-linearity of the Navier-Stokes equations and the unknown position of the interface. To compute its solution, we have carried out a linearization treating some terms semi-implicitly following the scheme proposed in [16]. Precisely, the convective term of the Navier-Stokes equations is treated explicitly and the level-set function is taken from the previous time step to compute the density and the viscosity. We introduce a time discretization $0=t_{0}<t_{1}<\cdots<t^{n}<t^{n+1}$ with uniform time step $\Delta t=t^{n+1}-t^{n}, \forall n$. Supposing that $\mathbf{u}^{n}, \phi^{n}, \rho^{n}$ and $\mu^{n}$ are known quantities at time $t^{n}$, we can compute the fluid velocity $\mathbf{u}^{n+1}$ and the pressure $p^{n+1}$ by solving the following:

$$
\begin{gathered}
\rho^{n}\left(\frac{1}{\Delta t} \mathbf{u}^{n+1}+\left(\mathbf{u}^{n} \cdot \nabla\right) \mathbf{u}^{n+1}\right)-\nabla \cdot \mathbf{T}\left(\mathbf{u}^{n+1}, p^{n+1} ; \mu^{n}\right)=\mathbf{f}^{n+1}+\frac{\rho^{n}}{\Delta t} \mathbf{u}^{n} \quad \text { in } \Omega \\
\nabla \cdot \mathbf{u}^{n+1}=0 \quad \text { in } \Omega
\end{gathered}
$$




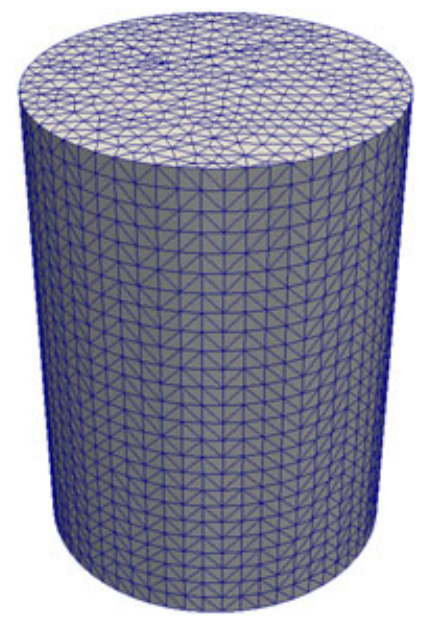

Figure 4. Mesh representing a 14.4-cm diameter cylinder used for all the simulations.

where $\mathbf{u}$ represents the global velocity $\left(\left.\mathbf{u}\right|_{\Omega_{\text {liquid }}}=\mathbf{u}_{l}\right.$ and $\left.\left.\mathbf{u}\right|_{\Omega_{\text {air }}}=\mathbf{u}_{a}\right), p$ the global pressure, f the global forcing term, $\rho$ the global density and $\mu$ the global viscosity ( $\rho$ and $\mu$ are therefore piecewise continuous functions with a discontinuity across $\Gamma$ ). After solving at each time step the linearized Navier-Stokes equations (15) and (16), we can compute the new location of the two fluids by solving the following transport-reaction equation:

$$
\mathbf{u}^{n+1} \cdot \nabla \phi^{n+1}+\frac{1}{\Delta t} \phi^{n+1}=\frac{1}{\Delta t} \phi^{n} \quad \text { in } \Omega
$$

and so, we can also update the density $\rho^{n+1}$ and the viscosity $\mu^{n+1}$.

In order to approximate the solutions of both the linearized Navier-Stokes equations (15) and (16) and the transport reaction equation (17), we introduce a mesh $\tau_{h}$ of the domain $\Omega$ (Figure 4), composed of tetrahedra $K$ (see [17] for an introduction to finite elements).

The level set function is discretized using $\mathbb{P}_{1}$ finite elements, that is, the discrete space $L_{h}$ in which we look for the approximation $\phi_{h}$ of $\phi$ is defined as

$$
L_{h}=\left\{\psi_{h} \in H^{1}(\Omega) \cap C^{0}(\bar{\Omega}):\left.\psi_{h}\right|_{K} \in \mathbb{P}_{1} \forall K \in \tau_{h}\right\} .
$$

However, the PDE governing the evolution of the level set function (17) is a transport-reaction equation and can therefore lead to numerical instabilities. An interior penalty stabilization [18] is then added to ensure the good behavior of the interface capturing, leading to the following weak formulation: find $\phi_{h}^{n+1} \in L_{h}$ such that

$$
\int_{\Omega} \mathbf{u}_{h}^{n+1} \cdot \nabla \phi_{h}^{n+1} \psi_{h}+\frac{1}{\Delta t} \int_{\Omega} \phi_{h}^{n+1} \psi_{h}+j_{\phi}\left(\phi_{h}^{n+1}, \psi_{h}\right)=\frac{1}{\Delta t} \int_{\Omega} \phi_{h}^{n} \psi_{h} \quad \forall \psi_{h} \in L_{h}
$$

with

$$
j_{\phi}\left(\phi_{h}, \psi_{h}\right)=\int_{\Gamma_{I}} \gamma_{\phi} h_{f}^{2}\left|\mathbf{u}_{h}^{n+1} \cdot \mathbf{n}\right| \llbracket \nabla \phi_{h} \rrbracket \llbracket \nabla \psi_{h} \rrbracket
$$

where $\Gamma_{I}$ represents the set of interior faces $\Gamma_{I}=\bigcup_{K} \partial K \backslash \partial \Omega, \gamma_{\phi}$ is a suitable coefficient, $\mathbf{n}$

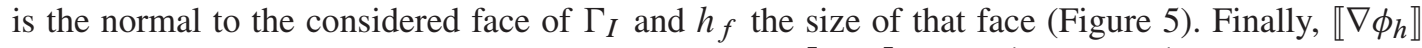
represents the jump of $\nabla \phi_{h}$ across the interface, that is $\llbracket \nabla \phi_{h} \rrbracket=\left.\nabla \phi_{h}\right|_{K_{1}}-\left.\nabla \phi_{h}\right|_{K_{2}}$ where $K_{1}$ and $K_{2}$ are the two elements adjacent to the considered face. This type of stabilization is particularly suitable for the level set advection as shown in [19].

Concerning the finite element approximation, for the linearized Navier-Stokes equations (15) and (16), we have chosen to use finite elements with bubble stabilization for the velocity $\mathbb{P}_{1}^{b}-\mathbb{P}_{1}$ in 


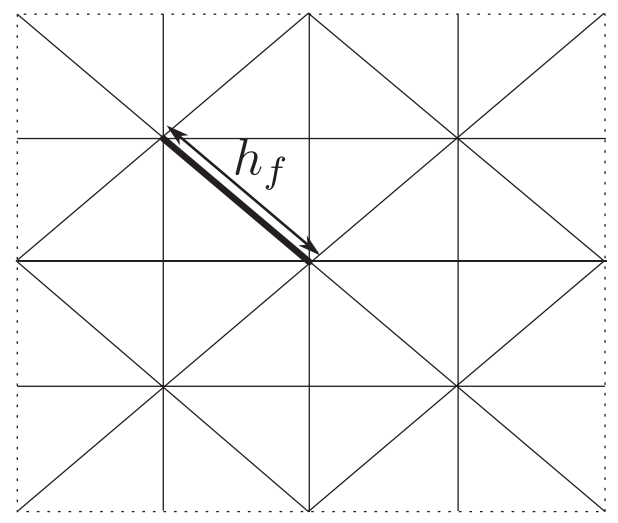

Figure 5. Two-dimensional representation of the geometrical entities involved in the IP stabilization: $\partial \Omega$ is represented by the dotted lines, $\Gamma_{I}$ by the normal lines and $h_{f}$ is indicated for the bold face.

order to fulfill the inf-sup condition [17]. Hence, the finite element space that we consider for the approximations $\mathbf{u}_{h}$ and $p_{h}$ of $\mathbf{u}$ and $p$, respectively, are $V_{h}^{3}$ and $P_{h}$ where

$$
\begin{gathered}
V_{h}=\left\{v_{h} \in H^{1}(\Omega) \cap C^{0}(\bar{\Omega}):\left.v_{h}\right|_{K} \in \mathbb{P}_{1}^{b} \forall K \in \tau_{h}\right\} \\
P_{h}=\left\{p_{h} \in C^{0}(\bar{\Omega}):\left.p_{h}\right|_{K} \in \mathbb{P}_{1} \forall K \in \tau_{h}\right\} .
\end{gathered}
$$

A definition of the space $\mathbb{P}_{1}^{b}$ can be found in [20]. The weak formulation corresponding to the linearized Navier-Stokes equations (15) and (16) is as follows: find $\mathbf{u}_{h}^{n+1} \in V_{h}^{3}$ and $\mathbf{p}_{h}^{n+1} \in P_{h}$ such that

$$
\begin{aligned}
& \int_{\Omega} \rho^{n}\left(\frac{1}{\Delta t} \mathbf{u}_{h}^{n+1}+\left(\mathbf{u}_{h}^{n} \cdot \nabla\right) \mathbf{u}_{h}^{n+1}\right) \cdot \mathbf{v}_{h} \\
&+\int_{\Omega} \frac{\mu^{n}}{2}\left(\nabla \mathbf{u}_{h}^{n+1}+\left(\nabla \mathbf{u}_{h}^{n+1}\right)^{T}\right):\left(\nabla \mathbf{v}_{h}+\nabla \mathbf{v}_{h}^{T}\right) \\
&-\int_{\Omega} p_{h}^{n+1} \nabla \cdot \mathbf{v}_{h} \\
&=\int_{\Omega} \mathbf{f}^{n+1} \cdot \mathbf{v}_{h}+\int_{\Omega} \frac{\rho^{n}}{\Delta t} \mathbf{u}_{h}^{n} \cdot \mathbf{v}_{h} \quad \forall \mathbf{v}_{h} \in V_{h}^{3} \\
& \int_{\Omega} \nabla \cdot \mathbf{u}_{h}^{n+1} q_{h}=0 \quad \forall q_{h} \in P_{h} .
\end{aligned}
$$

There is no boundary term because of the boundary conditions that we described in Section 2.1. In the weak formulations (20) and (21), we have not added stabilization terms to control the convection term. The reason is that, we will consider only cases where the viscous effects are dominating the convective ones. For other cases, for example, for fluids with low viscosity, one has to introduce numerical stabilization such as interior penalty [21] or SUPG [22].

\subsection{A pressure correction method}

One of the challenges in simulating the two-phase problems (20) and (21) arises from the large ratio of the densities across the free surface $\Gamma$. The first consequence is that standard quadrature procedures used in finite elements might lead to large errors in the integration of the terms involving the discontinuous density in the elements of the grid crossed by $\Gamma$. This problem can be overcome by resorting to quadrature rules that are adapted to the interface: in each element crossed by the interface, the position of the interface is computed (by finding the intersections of $\Gamma$ with the edges of the tetrahedron) and a quadrature rule is devised on each side by combining a few local quadrature rules (Figure 6). 


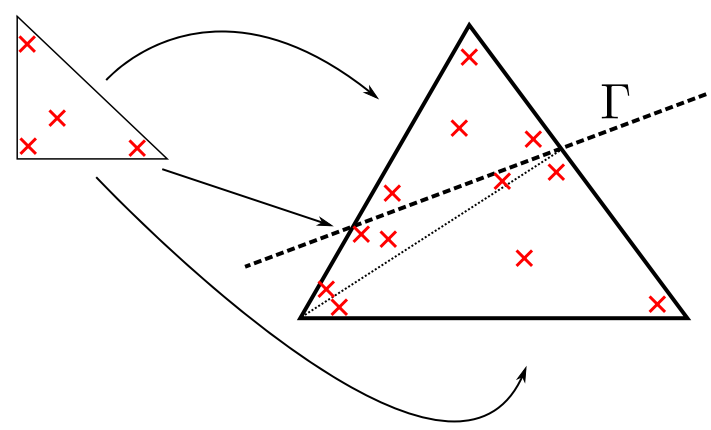

Figure 6. The quadrature rule in a triangle (bold lines) cut by the interface $\Gamma$ is built by triangulating (dotted line) both sides of the triangle, associating a quadrature rule to each of the triangles (arrows) and putting all the resulting quadrature points (crosses) together.

The second consequence of the large difference in the densities is that the approximation of the pressure can be very poor near the interface if one only relies on Lagrangian finite element spaces. Indeed, the pressure gradient is discontinuous across the interface as soon as the normal component of the force $\mathbf{f}$ in Equation (15) is discontinuous across the interface, as pointed out in [23]. In our case, these forces are discontinuous because of the presence of the density $\rho$ that is discontinuous across $\Gamma$. If we consider that the gravity is the dominating external force, we have the following:

$$
\llbracket \frac{\partial p}{\partial n} \rrbracket=\llbracket \rho \mathbf{g} \cdot \mathbf{n}_{\Gamma} \rrbracket=\llbracket \rho \rrbracket \mathbf{g} \cdot \mathbf{n}_{\Gamma} \equiv g_{n} .
$$

This discontinuity has to be captured if one wants to perform accurate simulations of experiments where $\llbracket \rho \rrbracket$ is large. Some techniques have already been developed to this purpose. A popular approach is to use XFEM methods to enrich the pressure space with basis functions that are discontinuous across the interface [24]. The main disadvantage is that additional degrees of freedom (DOFs) are added to the fluid problem. The method proposed in [6] overcomes this drawback by enriching the pressure space with basis functions that are both discontinuous across the interface and local to one element. A priori condensation is therefore performed, so that no additional DOFs appear in the final algebraic system.

In this paper, we will use a method inspired by [25] and [26] for solving elliptic problems with internal discontinuities. The main idea is to take advantage of the fact that the jump $\llbracket \frac{\partial p}{\partial n} \rrbracket$ is known, or approximately known if we neglect the fictitious forces. Indeed, in this method, an auxiliary function is built to account for the discontinuity and then used to build a problem for a regularized pressure. More precisely, we first note that the function $g_{n}$ can be extended easily to a function $\bar{g}_{n}=\llbracket \rho \rrbracket \mathbf{g} \cdot \nabla \phi$ defined on $\Omega$ by remarking that $\mathbf{n}_{\Gamma}=\nabla \phi$. We define then the auxiliary discrete function

$$
L_{p}(\mathbf{x})=\left\{\begin{array}{cc}
\sum_{\phi_{i}<0}\left(\bar{g}_{n}\left(\mathbf{x}_{i}\right) \phi\left(\mathbf{x}_{i}\right)\right) \varphi_{i}(\mathbf{x}) & \text { if } \phi(\mathbf{x}) \geqslant 0 \\
-\sum_{\phi_{i} \geqslant 0}\left(\bar{g}_{n}\left(\mathbf{x}_{i}\right) \phi\left(\mathbf{x}_{i}\right)\right) \varphi_{i}(\mathbf{x}) & \text { if } \phi(\mathbf{x})<0
\end{array}\right.
$$

where $\mathbf{x}_{i}$ is the position of the node associated to the basis function $\varphi_{i}$ of the discrete pressure space $P_{h}$. The idea behind this formula is that the jump of $L_{p}$ approximates the ones of the pressure $p$ :

$$
\llbracket L_{p} \rrbracket=\sum_{i}\left(\bar{g}_{n}\left(\mathbf{x}_{i}\right) \phi\left(\mathbf{x}_{i}\right)\right) \varphi_{i}(\mathbf{x}) \cong 0
$$

as $\left.\left(\sum_{i} \phi\left(\mathbf{x}_{i}\right) \varphi_{i}(\mathbf{x})\right)\right|_{\Gamma}=\left.\phi\right|_{\Gamma}=0$ and

$$
\llbracket \frac{\partial L_{p}}{\partial n} \rrbracket=\frac{\partial}{\partial n} \sum_{i}\left(\bar{g}_{n}\left(\mathbf{x}_{i}\right) \phi\left(\mathbf{x}_{i}\right)\right) \varphi_{i}(\mathbf{x}) \cong g_{n} .
$$


After being constructed, the lifting is subtracted from the pressure in order to form a regularized pressure $\hat{p}=p-L_{p}$, which is more regular, as we have

$$
\llbracket \hat{p} \rrbracket=\llbracket p \rrbracket-\llbracket L_{p} \rrbracket \cong 0
$$

and

$$
\llbracket \frac{\partial \hat{p}}{\partial n} \rrbracket=\llbracket \frac{\partial p}{\partial n} \rrbracket-\llbracket \frac{\partial L_{p}}{\partial n} \rrbracket \cong 0 .
$$

We can now rewrite the weak formulation of the linearized Navier-Stokes equations in terms of the new unknowns $\left(\mathbf{u}_{h}^{n+1}, \hat{p}_{h}^{n+1}\right)$ to be solved instead of the Equations (20) and (21):

$$
\begin{aligned}
& \int_{\Omega} \rho^{n}\left(\frac{1}{\Delta t} \mathbf{u}_{h}^{n+1}+\left(\mathbf{u}_{h}^{n} \cdot \nabla\right) \mathbf{u}_{h}^{n+1}\right) \cdot \mathbf{v}_{h} \\
&+\int_{\Omega} \frac{\mu^{n}}{2}\left(\nabla \mathbf{u}_{h}^{n+1}+\left(\nabla \mathbf{u}_{h}^{n+1}\right)^{T}\right):\left(\nabla \mathbf{v}_{h}+\nabla \mathbf{v}_{h}^{T}\right) \\
&-\int_{\Omega} \hat{p}_{h}^{n+1} \nabla \cdot \mathbf{v}_{h} \\
&=\int_{\Omega} \mathbf{f}^{n+1} \cdot \mathbf{v}_{h}+\int_{\Omega} \frac{\rho^{n}}{\Delta t} \mathbf{u}_{h}^{n} \cdot \mathbf{v}_{h}+\int_{\Omega} L_{p} \nabla \cdot \mathbf{v}_{h} \quad \forall \mathbf{v}_{h} \in V_{h}^{3} \\
& \int_{\Omega} \nabla \cdot \mathbf{u}_{h}^{n+1} q_{h}=0 \quad \forall q_{h} \in P_{h}
\end{aligned}
$$

where $L_{p}$ is the auxiliary function (23) built using $\phi=\phi_{h}^{n}$. The implementation of our method does not require heavy changes in the usual structure of a finite element code, as it only introduces a modification of the right hand side. Remark that no unknowns have been built during the construction of the additional right hand side, even if the lifting of the pressure $L_{p}$ actually belongs to an enriched pressure space.

To sum up, the algorithm was composed of four steps that were performed sequentially, at each time step:

1. Solve the linearized Navier-Stokes equations (28) and (29), where we used the interface position at the previous time step and the pressure correction scheme;

2. Update the location of the interface by advecting the level set function using problem (18);

3. Reinitialize the level set function: reconstruct the interface geometrically and redefine the level set as a distance function to the interface; and

4. Correct the volume of the different phases using (13).

\section{NUMERICAL RESULTS AND DISCUSSION}

To avoid numerical stabilization for the linearized Navier-Stokes problem and have faster computations, we restricted our investigation to a liquid having high viscosity. The considered aqueous solution is made of $98.5 \%$ glycerine (viscosity: $1 \mathrm{~Pa} \mathrm{~s}$ ).

For the numerical simulations, we used a mesh built out of tetrahedra, structured in the vertical direction. The mesh contained around 50,000 elements and 10,000 vertices. With the spatial discretization that we set, we got a total amount of around 190,000 DOFs for each linearized Navier-Stokes problem (180,000 for the velocity and 10,000 for the pressure) and 10,000 DOFs for each advection of the level set function.

For each linear system for the fluid problem, an additive Schwarz preconditioner [27] was built by the library Trilinos [28], using a complete $L U$ factorization for each subdomain. The same kind of preconditioner was used for the linear system associated with the level set advection problem. The whole code was based on the library LifeV (www.lifev.org). All the simulations were run on the Cray XT5 Rosa located at the Swiss National Supercomputing Centre, using 108 CPUs in order to get a similar final time for all the cases. 

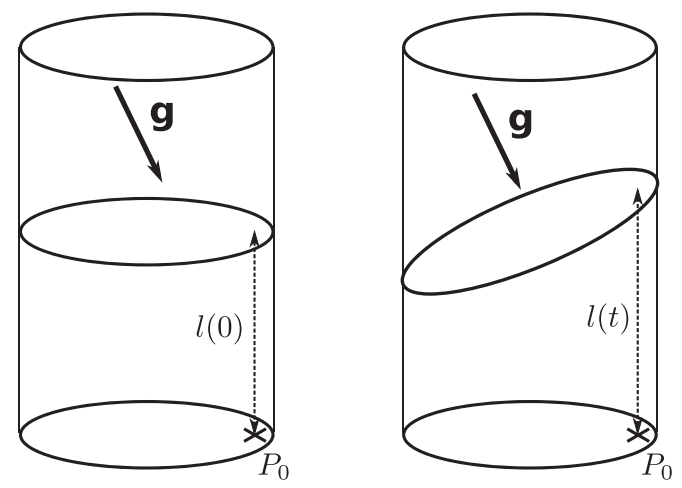

Figure 7. Schematic representation of the initial (left) and final (right) configurations in the tilted cylinder test.

\subsection{Tilted cylinder test}

To assess the quality of our method in simulating free surface flows, we devised a simple test consisting in putting the gravity off the vertical axis and in observing the motion of the free surface, without shaking the container. More precisely, the usual gravity field is replaced by

$$
\mathbf{g}=\left(\begin{array}{c}
g \sin \left(\alpha_{\text {tilt }}\right) \\
0 \\
-g \cos \left(\alpha_{\text {tilt }}\right)
\end{array}\right) .
$$

where $\alpha_{\text {tilt }}$ represents the angle between the gravity $\mathbf{g}$ and the $z$-axis. We used an angle $\alpha_{\text {tilt }}=\frac{\pi}{8}$. The cylinder had a diameter of $28.7 \mathrm{~cm}$, and the initial liquid height was $0.2 \mathrm{~m}$. A representation of the setting we are considering is shown in Figure 7.

We are interested in the oscillations of the free surface around its equilibrium position. The oscillations of the interface were tracked following the height $l(t)$ of the free surface above a reference point $P_{0}$ close to the wall, $P_{0}=(0.14,0,0)$ (Figure 7).

We tested several time steps in order to observe the influence of the time discretization. First of all, we could observe that the time step had to be smaller than $0.1 \mathrm{~s}$ for the scheme we used, otherwise, the free surface was not evolving towards a steady position, as shown in Figure 8.

Three other time steps $\Delta t=0.05,0.02,0.01$ were tested, and all of them gave satisfactory results as the surface evolved towards the equilibrium. The differences between the results obtained with the different time steps were small (Figure 9): with a smaller time step, the wave amplitude decreased slightly more quickly. A small phase shift could also be observed for large times (Figure 10).

Moreover, we remark that our pressure correction scheme is very effective in reducing the spurious velocities. Indeed, Figure 10 shows that oscillations of the free surface smaller than the cells size (their height is $0.02 \mathrm{~m}$ ) can be accurately captured, and that such oscillations remain smooth over long time intervals. This could not be possible if spurious currents would still be present. The pressure correction scheme developed in the previous section represents therefore a valuable and computationally cheap strategy.

\subsection{Wave amplitude in orbitally shaken bioreactors}

We consider the problem of simulating the flow inside the OSRs. The OSR considered in our simulations was a 14.4-cm diameter cylinder filled to a height of $0.1 \mathrm{~m}$ with an aqueous solution of $98.5 \%$ glycerine (viscosity: $1 \mathrm{~Pa} \mathrm{~s}$ ). Simulations were run for several agitation rates from 85 to $125 \mathrm{rpm}$ at a shaking radius of $2.5 \mathrm{~cm}$. On the basis of the tests carried out in the previous Section 5.1, for the time advancing, we used a uniform time step $\Delta t=0.05 \mathrm{~s}$ for all the simulations, and the calculations were run until a final time between 8 and $9 \mathrm{~s}$. 


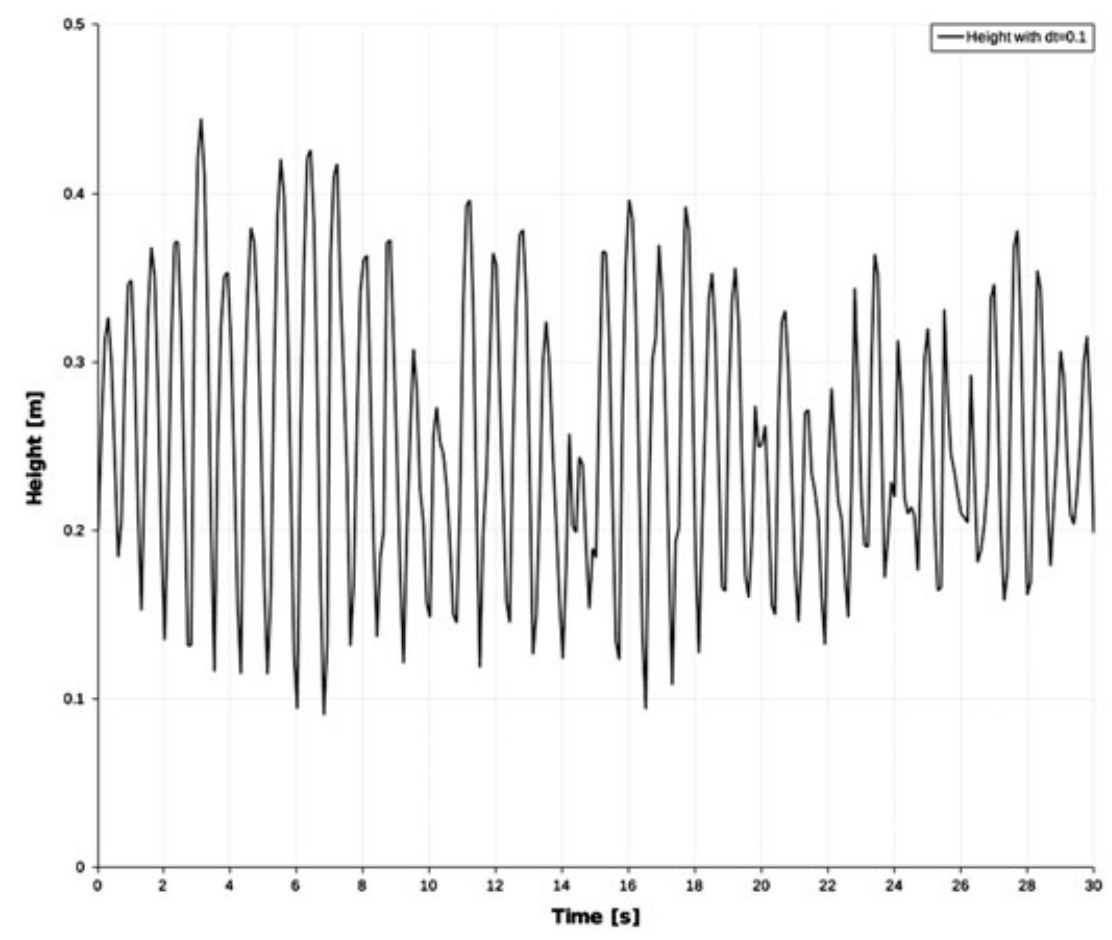

Figure 8. Evolution of $l(t)$ with a time step of $0.1 \mathrm{~s}$.

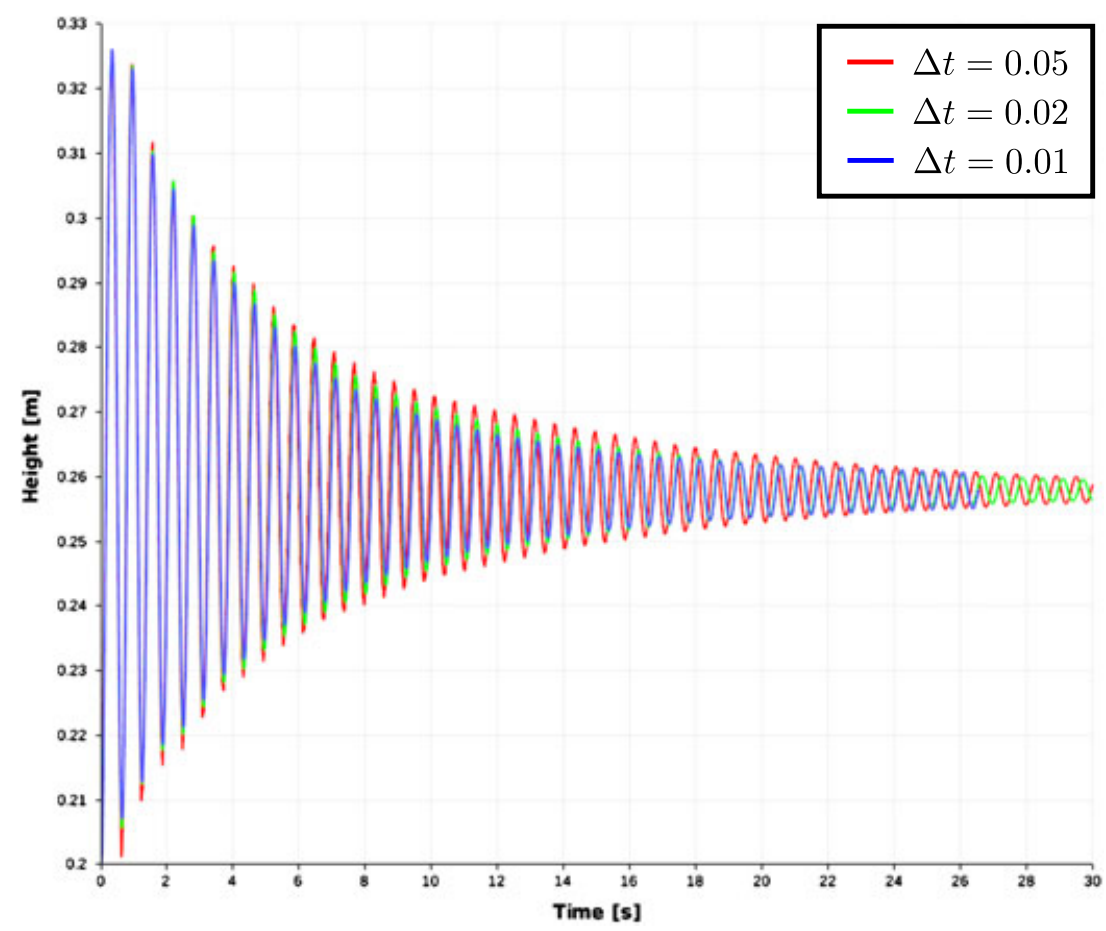

Figure 9. Evolution of $l(t)$ with time steps 0.05 (red), 0.02 (green) and 0.01 (blue).

The amplitude of the wave was the parameter that we used to compare our simulations with the experimental data. In the simulations, the amplitude of the wave was calculated by taking two vertical lines on the boundary of the cylinder and by tracing the liquid height on these two vertical 


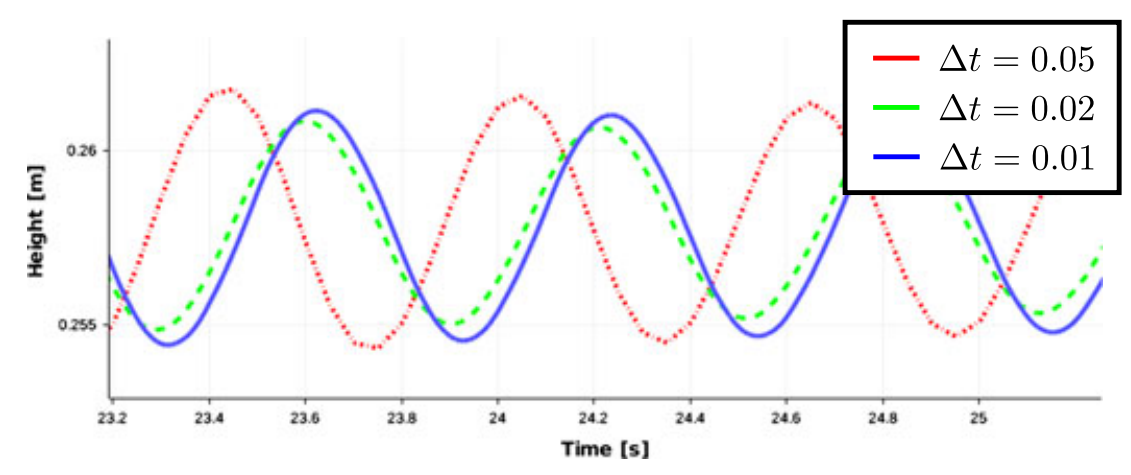

Figure 10. Zoom on Figure 9 to observe the evolution after a long period.

Time: $9.20(s)$
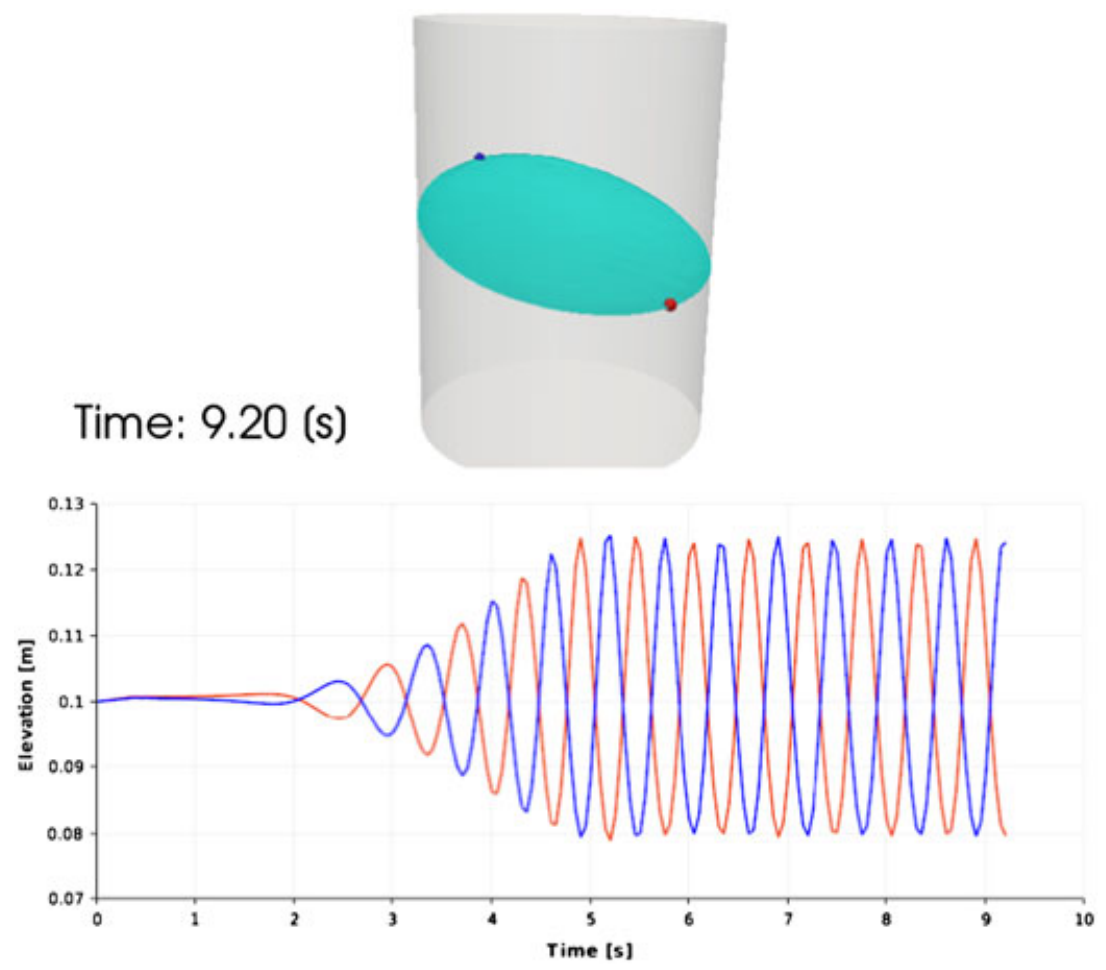

Figure 11. Schematic representation of the amplitude of the wave for a numerical simulation. The two vertical lines for the measure are represented by the blue and red spheres on the surface.

lines according to time. The maximal amplitude of the wave was read directly on the resulting graph (Figure 11).

All the conditions considered in our simulations were also experimentally tested. The experimental value of the amplitude of the wave was measured at the wall of the cylinder and was defined as the difference of liquid height between a crest and a trough.

In Figure 12, the amplitudes obtained by simulation with vertical slip were in good agreement with the experimental data at agitation rates from 85 to $105 \mathrm{rpm}$. At these agitation rates, the amplitude of the wave increased from 20 to $40 \mathrm{~mm}$. Between 115 and $125 \mathrm{rpm}$, the simulations showed larger amplitudes than the experimental data. With the vertical slip boundary conditions, the simulated amplitude increased by a factor 3 between 105 and $125 \mathrm{rpm}$, although experimental data showed a two-fold increase. 


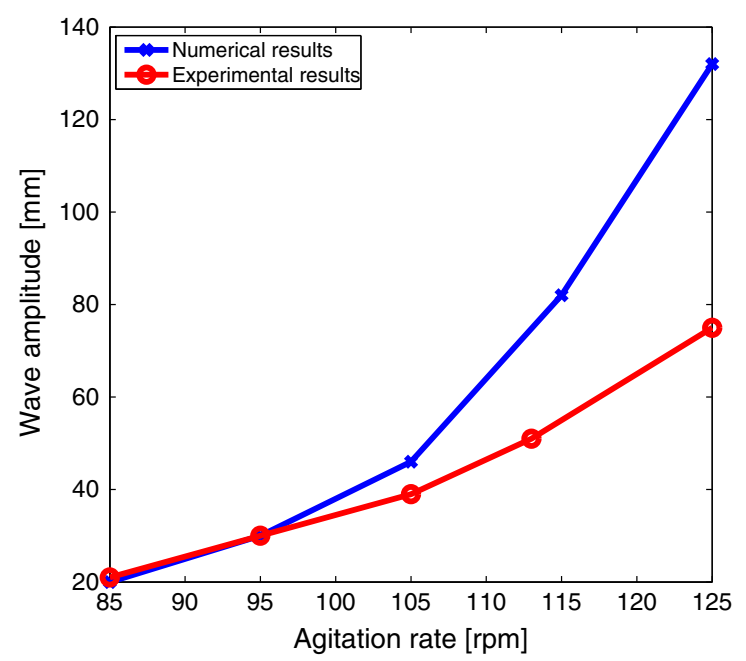

Figure 12. Maximal amplitude versus the agitation rate. Comparison between the experimental values (red) and the numerical values obtained with vertical slip boundary conditions (blue).

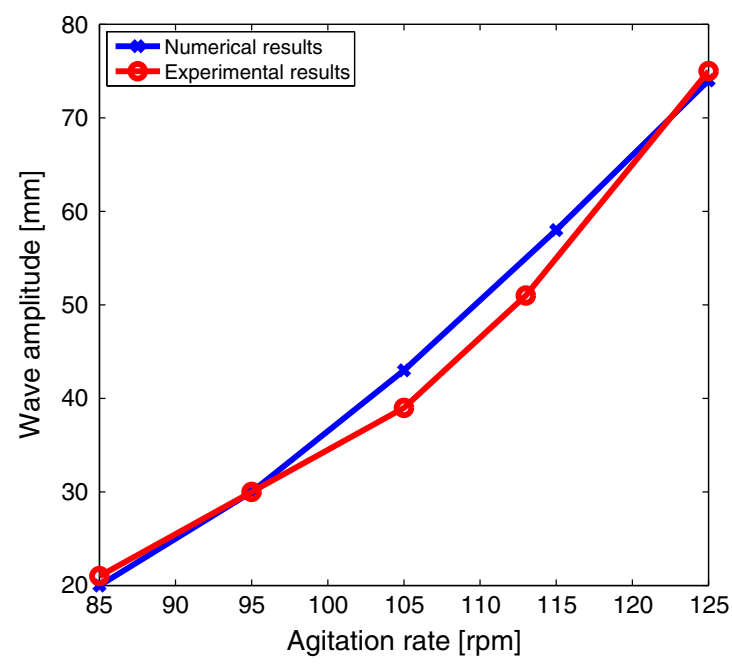

Figure 13. Maximal amplitude versus the agitation rate. Comparison between the experimental values (red) and the numerical values obtained with tangential slip boundary conditions (blue).

At $105 \mathrm{rpm}$, the slope of both the experimental and numerical curves increased, and a regime transition was observed (Figure 12). In hydrodynamics, this transition yields to a change of the liquid behavior, which was also captured during the simulations. At agitation rates lower than $105 \mathrm{rpm}$, the motion of the particles in the liquid is mainly local, whereas at higher agitation rates, larger displacements are observed in the liquid. The boundary conditions at the wall might not be suitable for the latter regime as all the power of the wave was channeled in the vertical direction pushing it higher than what it really should be.

The tangential slip boundary conditions allow the energy of the wave to be split in both the tangential and vertical directions preventing the wave to reach unphysical heights. Indeed, we observed during the simulations using these boundary conditions that the tangential velocity at the wall was not negligible in comparison with the vertical velocity when the agitation was higher than $105 \mathrm{rpm}$. As a consequence, the simulated amplitude with tangential slip increased by around two-fold between 105 and $125 \mathrm{rpm}$, which was in better agreement with the experimental data (Figure 13). 
The fact that the simulated amplitudes with both vertical and tangential slips gave similar results at agitation rates lower than $105 \mathrm{rpm}$ suggested that the boundary conditions did not affect the free surface at these agitation rates. However, as the agitation rate increased beyond $105 \mathrm{rpm}$, the choice of the boundary conditions became a critical aspect.

The shape of the interface was also considered as a benchmark for the simulations. Using the tangential slip boundary conditions, the interface was well captured at low agitation rates (Figure 14), whereas the computations were less accurate for higher agitation rates (Figure 15).

There may be two causes for the differences between the numerical and experimental interfaces. First, we did not take into account the friction losses at the wall, although experimentally, the interface was bent near the wall at $125 \mathrm{rpm}$, reflecting the friction between the wall and the liquid (Figure 15 (left)). An alternative to the problem would be to implement one of the boundary conditions proposed in [10] (where the same effects on the interface shape have been observed). The choice of a Robin boundary condition with coefficients adjusted by the level set function could be a good candidate. We will study this approach in Section 5.5. A second putative cause could be that at higher agitation rates, the fictitious forces caused by the motion of the bioreactor have a similar strength as gravity. The jump in the pressure gradient across the interface might therefore come from the discontinuity of that force across the interface. The pressure correction scheme should then take into account these effects.
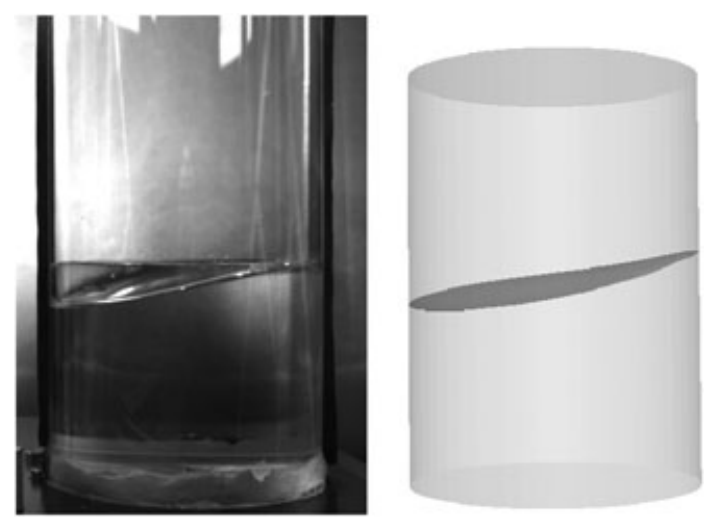

Figure 14. Interfaces of an aqueous solution of $98.5 \%$ glycerine at $95 \mathrm{rpm}$ captured during an experiment (left) and its numerical counterpart (right). The shaking radius was $2.5 \mathrm{~cm}$ and the liquid height $10 \mathrm{~cm}$.
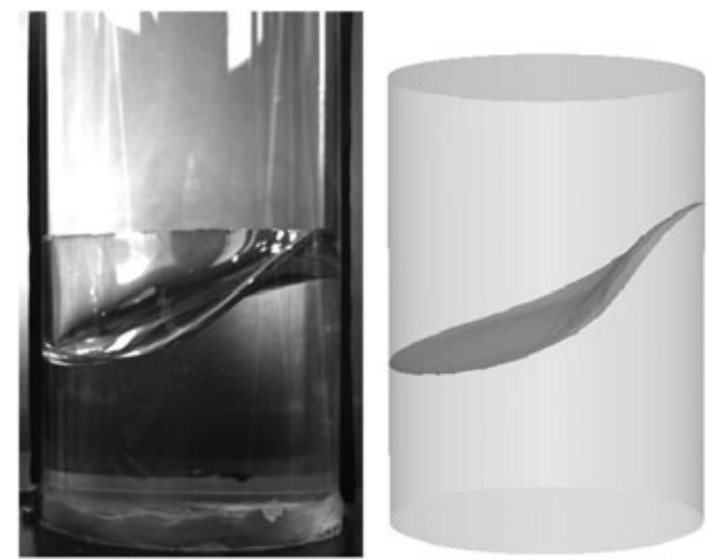

Figure 15. Interfaces of an aqueous solution of $98.5 \%$ glycerine at $125 \mathrm{rpm}$ captured during an experiment (left) and its numerical counterpart (right). The shaking radius was $2.5 \mathrm{~cm}$ and the liquid height $10 \mathrm{~cm}$. 
Table I. Maximal shear stress computed at the time $t=8 \mathrm{~s}$.

\begin{tabular}{lrrrrr}
\hline Agitation [rpm] & 85 & 95 & 105 & 115 & 125 \\
\hline Maximal shear stress [Pa] & 4000 & 7400 & 11900 & 29100 & 92500 \\
\hline
\end{tabular}

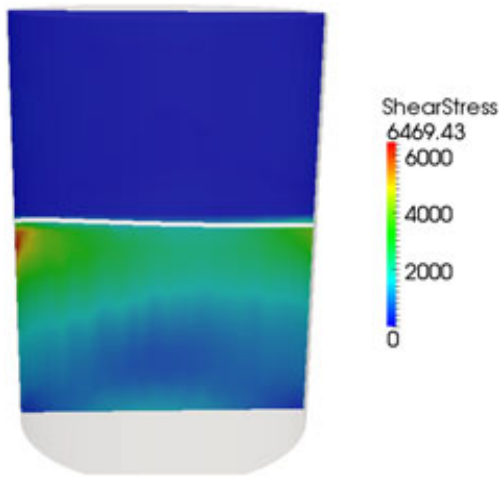

Figure 16. Shear stress magnitude induced by the shaking motion of $95 \mathrm{rpm}$ at time $t=8 \mathrm{~s}$ on a slice through the OSR. The free surface location is displayed by the white line.

\subsection{Shear stress calculations}

The evaluation of the shear stress magnitude and its localization in the bioreactor is important as it affects the viability of the cells in the culture. Indeed, high shear stress can disrupt the cell membrane, leading to cell death.

The simulations led to an approximation of the whole velocity field. We could deduce from it an approximated shear stress magnitude, here computed as $\mu\left\|\nabla \mathbf{u}+\nabla \mathbf{u}^{T}\right\|_{2}$ where $\|\cdot\|_{2}$ denotes the Frobenius norm, that is $\left\|\nabla \mathbf{u}+\nabla \mathbf{u}^{T}\right\|_{2}=\left(\sum_{i, j}\left(\partial_{i} u_{j}+\partial_{j} u_{i}\right)^{2}\right)^{1 / 2}$ (we computed this quantity in the whole bioreactor even if we were only interested in its value in the liquid phase $\Omega_{\text {liquid }}$ ). The influence of the boundary conditions used for the wall was very high in this case [10]: when the vertical slip was adopted, there was a high shear stress area near the walls, whereas when the tangential slip was used, the shear stress was lower. As the most relevant boundary condition for the liquid is the no-slip boundary condition and as the vertical slip is closer to that condition, we investigated the shear stress distribution only with the latter condition.

The shear stress increased quickly with the agitation rate (Table I). For agitation rates from 85 to $125 \mathrm{rpm}$, the shear stress was non-uniformly distributed: the higher shear stress levels were located near the wavefront (Figures 16 and 17). Because these calculations were performed using glycerol as the liquid phase, the shear stress level was assumed to be at least three orders of magnitude higher than in aqueous medium typically used for cell culture. We were then expecting lower shear stress levels than those reported to be lethal for mammalian cells at around $10^{2} \mathrm{~N} / \mathrm{m}^{2}$ [29].

\subsection{Mixing performance evaluation}

To evaluate the mixing capabilities of the OSRs, we calculated the pathway of particles on the basis of the computed liquid velocity. A series of 21 particles were introduced at each time step. They were placed in the vessel at 70,50 and $30 \mathrm{~mm}$ under the free surface and equally distributed in the radial direction (from the center of the vessel to $2 \mathrm{~mm}$ off the wall) and then passively advected by the fluid (as if they would have the same density as the liquid).

As we focused on the macromixing in OSRs, we neglected the influence of diffusion and considered that mixing occurred mainly through convection. At agitation rates lower than $105 \mathrm{rpm}$, and after $8 \mathrm{~s}$ of agitation, the particles underwent only a local motion, drawing spirals on the pictures (Figures 18 and 19). 


\section{SIMULATION OF ORBITALLY SHAKEN FLUIDS}

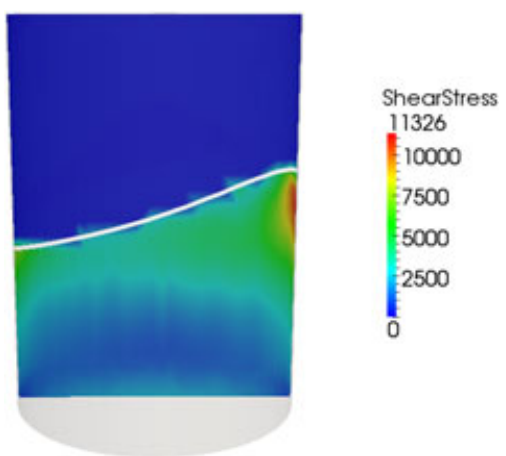

Figure 17. Shear stress magnitude induced by the shaking motion of $105 \mathrm{rpm}$ at time $t=8 \mathrm{~s}$ on a slice through the OSR. The free surface location is displayed by the white line.

Figure 18. Particle distribution viewed from below at $t=8 \mathrm{~s}$ for an agitation rate of $85 \mathrm{rpm}$ (particles are colored with respect to the radial coordinate of the release location).

Figure 19. Particle distribution viewed from below at $t=8 \mathrm{~s}$ for an agitation rate of $95 \mathrm{rpm}$ (particles are colored with respect to the radial coordinate of the release location). 
At agitation rates equal to or greater than $105 \mathrm{rpm}$, the global displacement of the particles was larger (Figures 20 and 21). At $115 \mathrm{rpm}$, the particles were disbursed within the bioreactor.

The transition between the two mixing regimes at $105 \mathrm{rpm}$ correlated to the one observed with the interface. The second regime was more favorable in terms of mixing of the different layers, and therefore, to the distribution of the oxygen in the liquid phase. These regime transitions were also observed in a previous study on the mixing principles in OSRs [30].

\subsection{A first attempt to use Robin boundary conditions}

The two proposed sets of boundary conditions used so far presented the following drawbacks:

1. Vertical slip could not reproduce the motion of the free surface at high agitation rates.

2. Tangential slip prevents the computation of correct wall shear stress.

Figure 20. Particle distribution viewed from below at $t=8 \mathrm{~s}$ for an agitation rate of $105 \mathrm{rpm}$ (particles are colored with respect to the radial coordinate of the release location).

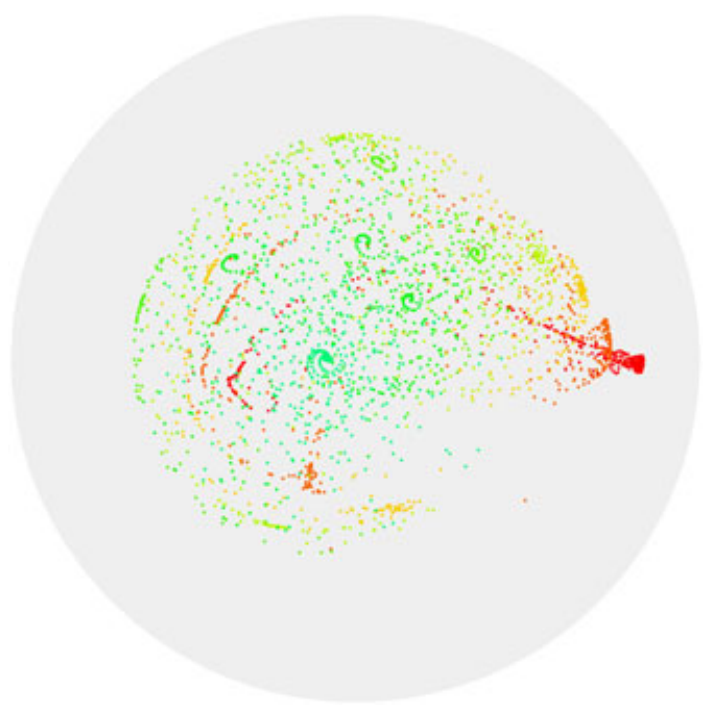

Figure 21. Particle distribution viewed from below at $t=8 \mathrm{~s}$ for an agitation rate of $115 \mathrm{rpm}$ (particles are colored with respect to the radial coordinate of the release location). 
We present, here, preliminary results using Robin-like boundary conditions as suggested in [10]. Robin boundary conditions consist in imposing, at the wall of the cylinder where the contact line is moving, the no-penetration condition

$$
\mathbf{u} \cdot \mathbf{n}=0 \quad \text { on } \Gamma_{\text {wall }}
$$

with two additional Robin conditions

$$
\begin{array}{cc}
S(\phi) \mathbf{u} \cdot \tau+(\mathbf{T}(\mathbf{u}, p ; \mu) \mathbf{n}) \cdot \tau=0 & \text { on } \Gamma_{\text {wall }} \\
S(\phi) \mathbf{u} \cdot \mathbf{e}_{z}+(\mathbf{T}(\mathbf{u}, p ; \mu) \mathbf{n}) \cdot \mathbf{e}_{z}=0 & \text { on } \Gamma_{\text {wall }}
\end{array}
$$

where $S(\phi)$ is a function of the level set used to weight the different components of the condition. Indeed, we ideally want that

- near the interface, a slip condition should be recovered, so $S(0)$ should be small; and

- far from the interface, a no-slip condition is the most suitable choice. For large values of $\phi$, $S(\phi)$ should be large to mimic the no-slip condition.

A simple candidate for $S$ is given by

$$
S(\phi)=\left\{\begin{array}{cl}
\mu(\phi) K & \text { if }|\phi| \geqslant s_{l} \\
0 & \text { if }|\phi|<s_{l}
\end{array}\right.
$$

where $K$ is a large constant (we used $10^{4}$ ) and $s_{l}$ is a parameter to be chosen, called slip length. Remark the presence of the viscosity $\mu$ in (31), which was found to better balance the amount of slip between the two phases.

We used Robin conditions with the tilted cylinder test described in Section 5.1. The slip length was the most important parameter (Figures 22 and 23): a too small slip length introduces a large damping of the surface motion and makes it adhere to the walls in an unphysical way. On the opposite, a large slip length would simply reduce the effect of the Robin condition with respect to the

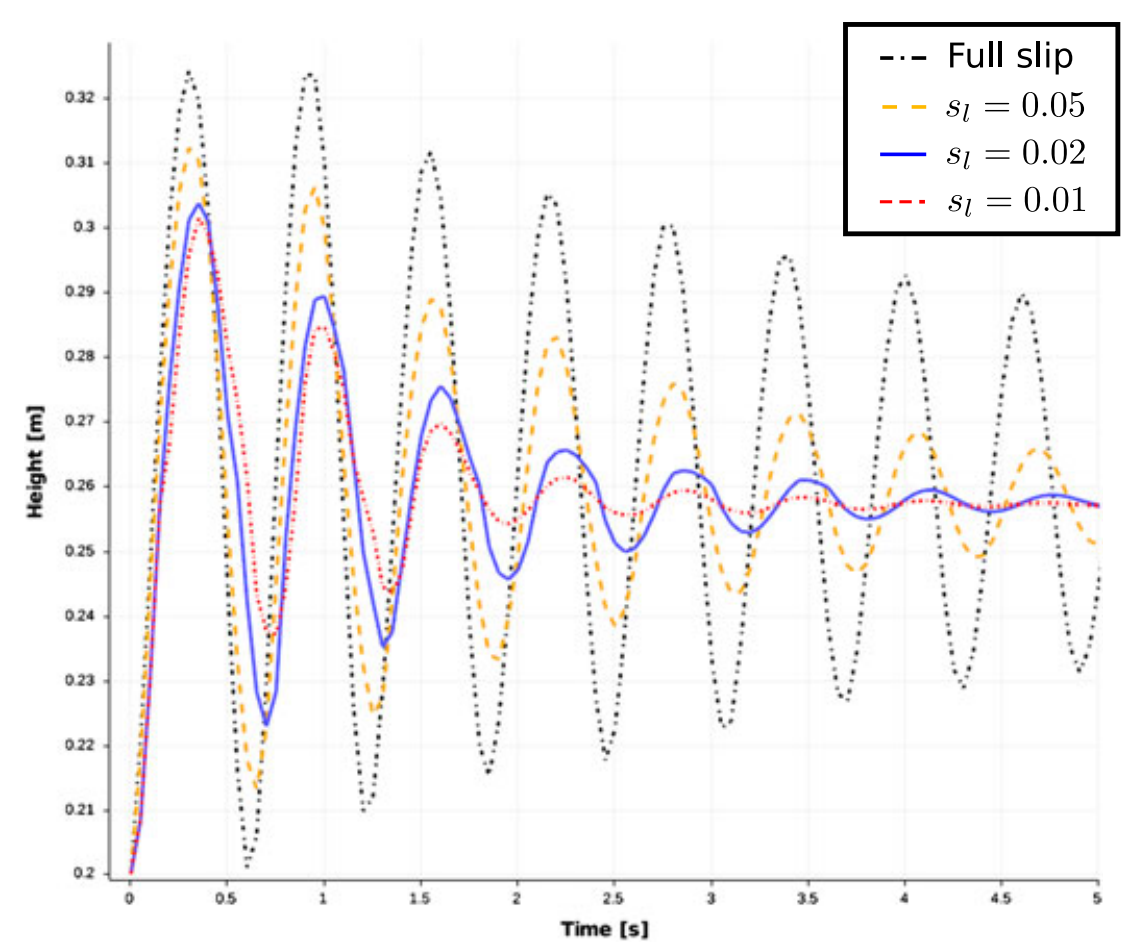

Figure 22. Free surface height for the tilted cylinder test case for different boundary conditions. 


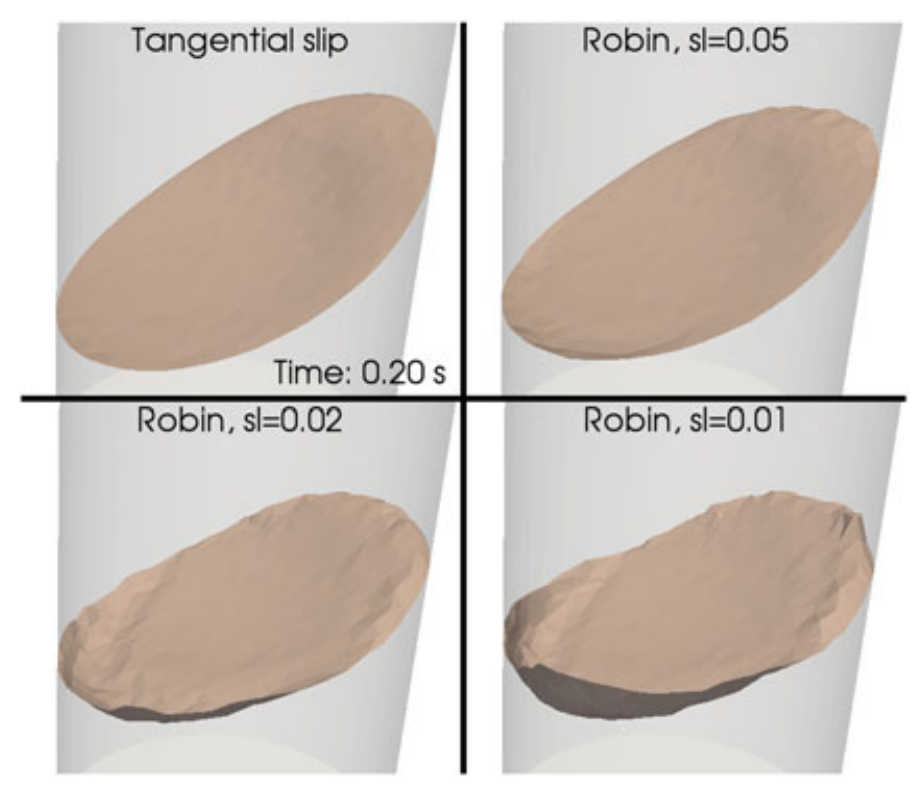

Figure 23. Different shapes of the free surface observed at time $0.2 \mathrm{~s}$ for the tilted cylinder test case, using different boundary conditions.

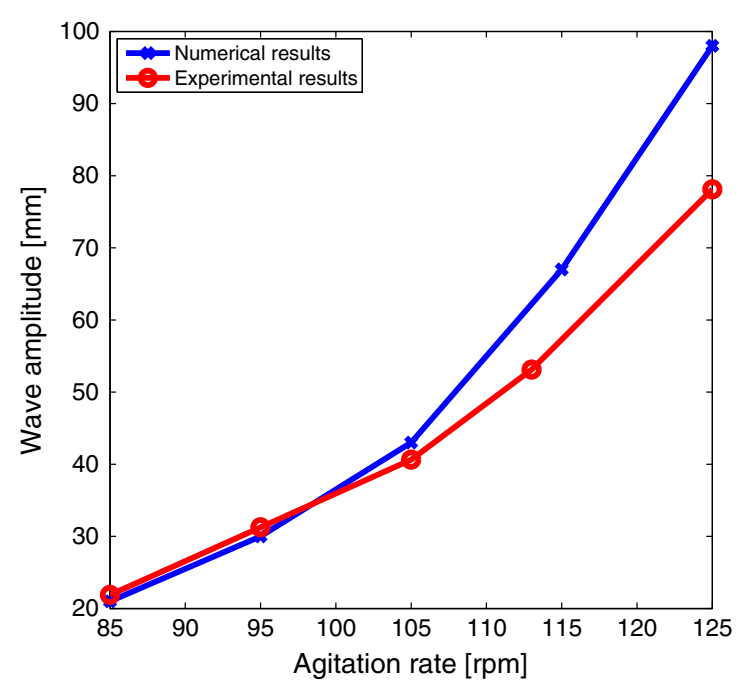

Figure 24. Comparison of the amplitude of the wave in the orbitally shaken bioreactors measured and computed numerically with Robin boundary condition.

tangential slip condition. Minimal slip lengths to avoid unphysical results were found between 0.05 and 0.1 . Note that these values might depend strongly on the mesh used.

We conducted the test with the wave amplitude measurements presented in Section 5.2 using Robin conditions and a slip length of 0.04 (which would correspond to a slip length of 0.08 for the tilted cylinder test, as there is a factor 2 between the two geometries).

The amplitude of the wave computed using this Robin boundary condition represented an intermediate case between those found with tangential and vertical slip conditions. Indeed, the amplitude was still overestimated for high agitation rates (above $105 \mathrm{rpm}$ ), but much less than with vertical slip (compare Figure 24 with Figures 12 and 13).

Even if the results could be probably improved by tuning the transition function $S(\phi)$ more finely, in particular, the slip length $s_{l}$, this test shows that Robin boundary conditions can give reasonable results for the interface motion. 


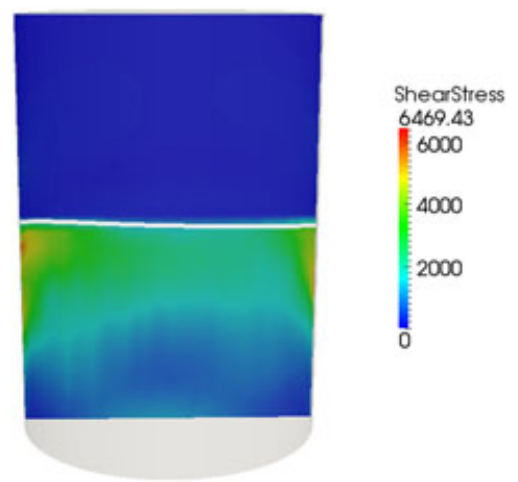

Figure 25. Shear stress magnitude induced by the shaking motion at $95 \mathrm{rpm}$ at time $t=8 \mathrm{~s}$ on a vertical section of the orbitally shaken bioreactor when Robin boundary conditions are used. The free surface location is displayed by the white line. This figure should be compared with Figure 16.

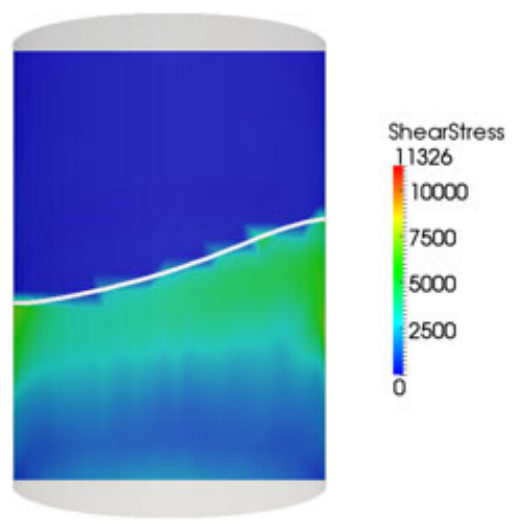

Figure 26. Shear stress magnitude induced by the shaking motion at $105 \mathrm{rpm}$ at time $t=8 \mathrm{~s}$ on a vertical section of the orbitally shaken bioreactor when Robin boundary conditions are used. The free surface location is displayed by the white line. This figure should be compared with Figure 17.

We also performed the same shear stress computations as in Section 5.3. The results are shown in Figures 25 and 26.

We can observe that Robin boundary conditions yield a lower maximal shear stress. Indeed, the maximal shear stress is usually located near the interface and Robin boundary conditions allow the fluids to fully slip in that area, preventing high shear stress. However, outside the slip bandwidth, shear stress with or without Robin boundary conditions are comparable in magnitude, Robin boundary conditions usually giving higher values.

Robin boundary conditions represent a good choice for the simulation of both the interface shape and the shear stress field, opposite to vertical and tangential slip conditions. The only drawback of the Robin boundary conditions is the necessity to finely tune the parameters, in particular the slip length $s_{l}$, which might be a non-trivial task.

\section{CONCLUSION}

Orbital shaking for suspension cultures of animal cells is, at least for large-scale applications, an entirely new approach to achieve efficient mixing and gas transfer without excessive hydrodynamic stress. Because mixing and gas transfer are increasingly more difficult problems from an engineering point of view for large-scale bioreactors, a mathematical approach to begin to understand air/liquid interphase conditions through modeling was considered.

In this paper, we showed a new method to deal with two-phase problems, where the difference in flow density is large. Spurious velocities did not appear near the interface and therefore did not 
pollute the solution, leading to physically meaningful surface shapes. This method was not computationally expensive, as it only changed the right hand side of the momentum equation and did not introduce additional DOFs.

The simulations gave us indications about the hydrodynamics in an OSR, where two mixing regimes were observed. The first regime, present only at low agitation rates, induced only local mixing and is expected to lead to poor oxygenation of the liquid phase. At agitation rates higher than $105 \mathrm{rpm}$ (for the 'scale of operation' studied here), improved mixing that correlated with the observed mixing efficiency of OSRs [30] was observed.

These two regimes should be investigated at water-like viscosities in order to better match the conditions of OSRs. However, this will require more advanced techniques for simulating the fluid flow, such as numerical stabilization or turbulence modeling. The boundary conditions should also be more deeply investigated. Indeed, the tangential slip condition gave relatively good results for the wave amplitude, but its inability to give correct shear stress values represents a significant problem. Robin boundary conditions represented a very satisfactory alternative. However, further investigations will be carried out to exploit them at best choosing in an optimal way the parameters involved in their definition. These conditions will be considered in further modeling studies of OSRs containing water, thus providing important insights that are expected to guide the development of efficient OSRs for large-scale cell culture operations.

\section{ACKNOWLEDGEMENTS}

The authors would like to thank the LMH laboratory of the EPFL, especially Dr M. Farhat, M. Reclari and G. Hannema for providing the experimental results used in this paper. This research was supported by the Swiss National Science Foundation through the project Sinergia 'Fluid dynamics and mixing behavior in orbitally shaken bioreactors for mammalian cell cultivation' (project n. CRSII2-125444). The Swiss National Supercomputing Centre is also acknowledged for providing computational resources. The authors also thank the anonymous reviewers for their very useful remarks. Swiss National Science Foundation CRSII2-125444.

\section{REFERENCES}

1. Muller N, Girard P, Hacker DL, Jordan M, Wurm FM. Orbital shaker technology for the cultivation of mammalian cells in suspension. Biotechnol. Bioeng. 2005; 89(4):400-406.

2. Stettler M. Bioreactor processes based on disposable materials for the production of recombinant proteins from mammalian cells. Ph.D. Thesis, 2007. EPFL.

3. Stettler M, Zhang X, Hacker DL, De Jesus M, Wurm FM. Novel orbital shake bioreactors for transient production of CHO derived IgGs. Biotechnology Progress 2007; 23(6):1340-1346.

4. Zhang X, Stettler M, Sanctis D, Perrone M, Parolini N, Discacciati M, De Jesus M, Hacker DL, Quarteroni A, Wurm FM. Use of orbital shaken disposable bioreactors for mammalian cell cultures from the milliliter-scale to the 1,000-liter scale. Advances in Biochemical Engineering/Biotechnology 2010; 115:33-53.

5. Lara AR, Galindo E, Ramírez OT, Palomares LA. Living with heterogeneities in bioreactors: understanding the effects of environmental gradients on cells. Molecular biotechnology 2006; 34(3):355-381.

6. Coppola-Owen AH, Codina R. Improving Eulerian two-phase flow finite element approximation with discontinuous gradient pressure shape functions. International journal for numerical methods in fluids 2005; 49:1287-1304.

7. Chessa J, Belytschko T. An enriched finite element method and level sets for axisymmetric two-phase flow with surface tension. International Journal for Numerical Methods in Engineering 2003; 58(13):2041-2064.

8. Gross S. Pressure XFEM for two-phase incompressible flows with application to 3D droplet problems. In Meshfree Methods for Partial Differential Equations V, Vol. 79, Griebel M, Schweitzer MA (eds), Lecture Notes in Computational Science and Engineering. Springer: Berlin, Heidelberg, 2011; 81-87.

9. Zunino P, Cattaneo L, Colciago CM. An unfitted interface penalty method for the numerical approximation of contrast problems. Applied Numerical Mathematics 2011; 61(10):1059-1076.

10. Robertson I, Sherwin SJ, Graham JMR. Comparison of wall boundary conditions for numerical viscous free surface flow simulation. Journal of Fluids and Structures 2004; 19(4):525-542.

11. Engelman MS, Sani RL, Gresho PM. The implementation of normal and/or tangential boundary conditions in finite element codes for incompressible fluid flow. International journal for numerical methods in fluids 2005; 2(3):225-238.

12. Sethian JA. Level Set Methods and Fast Marching Methods, (2nd edn). Cambridge University Press: Cambridge, 1999.

13. Sussman M, Puckett EG. A coupled level set and volume-of-fluid method for computing 3D and axisymmetric incompressible two-phase flows. Journal of Computational Physics 2000; 162(2):301-337. 
14. Enright D, Fedkiw R, Ferziger J, Mitchell I. A hybrid particle level set method for improved interface capturing. Journal of Computational Physics 2002; 183(1):83-116.

15. Smolianski A. Finite-element/level-set/operator-splitting (FELSOS) approach for computing two-fluid unsteady flows with free moving interfaces. International journal for numerical methods in fluids 2005; 48(3):231-269.

16. Winkelmann C. Interior penalty finite element approximation of Navier-Stokes equations and application to free surface flows. Ph.D. Thesis, Ecole Polytechnique Fédérale de Lausanne, 2007.

17. Quarteroni A. Numerical Models for Differential Problems. Springer: Milan, 2009.

18. Burman E, Hansbo P. Edge stabilization for Galerkin approximations of convection-diffusion-reaction problems. Computer Methods in Applied Mechanics and Engineering 2004; 193(15-16):1437-1453. Recent Advances in Stabilized and Multiscale Finite Element Methods.

19. Di Pietro DA, Lo Forte S, Parolini N. Mass preserving finite element implementations of the level set method. Applied Numerical Mathematics 2006; 56(9):1179-1195. Numerical Methods for Viscosity Solutions and Applications.

20. Quarteroni A, Valli A. Numerical Approximation of Partial Differential Equations. Springer: Berlin, 1994.

21. Burman E, Fernandez MA, Hansbo P. Continuous interior penalty finite element method for Oseen's equations. SIAM journal on numerical analysis 2006; 44(3):1248-1274.

22. Brooks AN, Hughes TJR. Streamline upwind/Petrov-Galerkin formulations for convection dominated flows with particular emphasis on the incompressible Navier-Stokes equations. Computer Methods in Applied Mechanics and Engineering 1982; 32(1-3):199-259.

23. Li Z, Lai M-C. The immersed interface method for the Navier-Stokes equations with singular forces. Journal of Computational Physics 2001; 171(2):822-842.

24. Belytschko T, Moes N, Usui S, Parimi C. Arbitrary discontinuities in finite elements. International Journal for Numerical Methods in Engineering 2001; 50(4):993-1013.

25. Huh J-S, Sethian JA. Exact subgrid interface correction schemes for elliptic interface problems. PNAS 2008; 105(29):9874-9879.

26. Discacciati M, Quarteroni A, Quinodoz S. Numerical approximation of internal discontinuity interface problems. Technical Report 18.2010, EPFL-MATHICSE, 2010.

27. Quarteroni A, Valli A. Domain Decomposition Methods for Partial Differential Equations. Oxford University Press, 1999.

28. Sala M, Heroux M. Robust algebraic preconditioners with IFPACK 3.0. Tachnical Report SAND-0662, Sandia National Laboratories, 2005.

29. Ma N, Koelling KW, Chalmers JJ. Fabrication and use of a transient contractional flow device to quantify the sensitivity of mammalian and insect cells to hydrodynamic forces. Biotechnology and Bioengineering 2002; 80(4):428-437.

30. Tissot S, Farhat M, Hacker DL, Anderlei T, Kühner M, Comninellis C, Wurm FM. Determination of a scale-up factor from mixing time studies in orbitally shaken bioreactors. Biochemical Engineering Journal 2010; 52:181-186. 